Revista Destaques Acadêmicos, Lajeado, v. 11, n. 3, 2019. ISSN 2176-3070

DOI: http://dx.doi.org/10.22410/issn.2176-3070.v11i3a2019.2269

http://www.univates.br/revistas

\title{
ATENDIMENTO ODONTOLÓGICO A HIPERTENSOS E DIABÉTICOS NA ATENÇÃO PRIMÁRIA À SAÚDE
}

\author{
Cosmo Helder Ferreira da Silva ${ }^{1}$, Adricia Kelly Marques Bento ${ }^{2}$, \\ Luiz Filipe Barbosa Martins ${ }^{3}$, Ana Caroline Rocha de Melo Leite ${ }^{4}$, \\ Vânia Barbosa do Nascimento ${ }^{5}$
}

\begin{abstract}
Resumo: No Brasil, o aumento da longevidade ocasionou uma maior probabilidade de desenvolvimento de doenças crônicas devido a mudanças na qualidade de vida dos idosos, por vezes prejudicada pela falta de informação ou até mesmo de incentivo. $\mathrm{O}$ cirurgião dentista tem papel importante no cuidado desses pacientes, pois através do acompanhamento odontológico é possível disponibilizar uma melhoria na saúde de forma geral. O presente estudo objetivou identificar o conhecimento dos profissionais de Odontologia da atenção primária à saúde no município de Quixadá-CE, frente ao tratamento odontológico de pacientes hipertensos e diabéticos. Trata-se de um estudo transversal de natureza descritiva e quantitativa de amostra não probabilística realizado com cirurgiões dentistas da sede e dos distritos. Os dados foram coletados através de um questionário estruturado entre o mês de março e abril de 2019. Os dados foram analisados de forma descritiva, categorizados, dicotomizados e submetidos à análise estatística por meio do SPSS Statistics 25.0. Participaram do estudo todos os 15
\end{abstract}

1 Cirurgião-dentista, Docente do Curso de Odontologia do Centro Universitário Católica de Quixadá (UNICATÓLICA), Quixadá-Ceará, Pós-Graduado em Saúde da Família e Doutorando em Ciências da Saúde no Cento Universitário Saúde ABC (CUSABC), Santo André-São Paulo, E-mail: helderferreira_18@yahoo.com.br

2 Cirurgiã-dentista, Centro Universitário Católica de Quixadá (UNICATÓLICA), QuixadáCeará, E-mail: adriciamarques@hotmail.com

3 Cirurgião-dentista, Docente do Curso de Odontologia do Centro Universitário Católica de Quixadá, (UNICATÓLICA), Doutor em Odontopediatria pela Faculdade de Odontologia de Piracicaba, Universidade Estadual de Campinas (FOP-UNICAMP), Campinas, São Paulo, E-mail: luizfilipe@unicatolicaquixada.edu.br

4 Cirurgiã-dentista, Docente do Curso de Enfermagem da Universidade da Integração Internacional da Lusofonia Afro-Brasileira (UNILAB), Doutora em Ciências Médicas pela Universidade Federal do Ceará (UFC), Fortaleza, Ceará, E-mail: acarolmelo@unilab.edu.br

5 Médica, Docente do Centro Universitário Saúde ABC (CUSABC), Doutora em Medicina Preventiva e Social pela Universidade de São Paulo (USP), São Paulo, E-mail: vaniabn@uol.com.br 
cirurgiões dentistas da atenção básica à saúde, sendo $86,7 \%(n=15)$ do sexo feminino e $13,3 \%(n=2)$ do sexo masculino. Dentre eles, 66,7\% $(n=10)$ atuam na Odontologia há $\leq$ 9 anos. Todos os entrevistados realizam acompanhamento odontológico, $100 \%(n=15)$. Porém, 46,7\% ( $n=7)$ não solicitam exames complementares. 46,7\% (n=7) observam resistência dos pacientes para realização do tratamento. Conclui-se a necessidade de capacitação dos profissionais para a padronização dos atendimentos e promoção de saúde para os pacientes terem conhecimento da importância de serem acompanhados por um cirurgião-dentista.

Palavras-chave: Cuidados primários. Doenças Crônicas. Assistência Odontológica.

\section{INTRODUÇÃO}

O declínio nas taxas de mortalidade fez com que houvesse um processo de envelhecimento populacional, realidade encontrada mundialmente. Porém, o processo não trouxe apenas benefícios como o aumento da longevidade. A ocorrência de um perfil de morbimortalidade foi ampliada devido ao aumento de doenças crônicas degenerativas (GOMES et al., 2013).

A qualidade de vida de idosos é afetada devido a patologias que podem influenciar diretamente na redução de sua autonomia. O acompanhamento com profissionais da saúde é indispensável para evitar a evolução dessas doenças, pois com o avanço da idade elas podem causar a incapacidade da população afetada (MIYAMOTO et al., 2016).

Doenças crônicas têm um grande impacto na vida das pessoas afetadas e de todos aqueles que estão em sua convivência. Por ter implicações graves, são doenças que podem levar a um alto índice de óbito, incapacidades e efeitos econômicos adversos.. As mesmas necessitam de atenção e cautela por conta dos problemas que podem ocasionar, como a insuficiência renal, mutilação de membros, deficiência visual (podendo levar a cegueira), alterações cardiovasculares, encefálicas, coronárias e vasculares periféricas (ALVES; BARBOSA, 2010).

A Estratégia de Saúde da Família (ESF) foi implantada no Brasil com o intuito de proporcionar melhoria para o serviço público. Ela é caracterizada para a Atenção Primária (AP) como prioritária na ampliação, concretização e qualificação do acesso aos serviços de saúde. Isso resulta no fortalecimento do atendimento, pois sua resolubilidade proporciona grande impacto na saúde de toda a comunidade atendida (BRASIL, 2016).

As equipes de ESF estão preparadas para oferecer à população um atendimento destinado à promoção, proteção e recuperação da saúde em nível de atenção primária. São atividades desenvolvidas pelas equipes multiprofissionais, responsáveis pelo desenvolvimento de atividades voltadas para cada público, em que os idosos contam com um acompanhamento e assistência especial que priorizam a qualidade de vida (MOTTA; AGUIAR; CALDAS, 2011). 
Através do vínculo entre a equipe e os usuários dos serviços de saúde é possível encadear os conceitos de humanização, integralidade do cuidado, além da responsabilidade dos serviços, resultando assim na aproximação em geral dos indivíduos, sendo de grande importância para o favorecimento e desenvolvimento do cuidado por meio de uma confiança, dedicação e partilha (ILHA et al., 2014).

A aproximação da equipe com a população é uma importante estratégia para o cuidado e prática profissional no que se refere a reorganização da assistência de saúde destinados ao paciente, possibilitando e garantindo o direito ao acesso, ao atendimento humanizado e solução dos serviços (BACKES et al., 2015).

A Política de Saúde Bucal do Brasil recomenda ações, as quais, juntamente com a atenção básica, devem ser discutidas e aplicadas, proporcionando uma integralidade no atendimento, de maneira que amplie e favoreça a demanda que utiliza os serviços públicos (CHAVES et al., 2010).

O mercado de trabalho odontológico é renovado a cada ano que passa por milhares de dentistas. No entanto, as diferentes escolas de ensino proporcionam níveis de conhecimento diferentes. Devido a essa variabilidade, é necessária a realização de reciclagem profissional, voltada principalmente aos cuidados de saúde geral, impedindo a interferência no acompanhamento odontológico (FABRIS et al., 2018).

A necessidade de um acompanhamento a pacientes portadores de hipertensão e diabetes torna-se cada vez mais necessária, visto o aumento da população mundial que necessita de atenção especial. O cirurgião dentista, como membro da ESF, assume um papel fundamental para o tratamento desses pacientes. Acredita-se que, mesmo com o incentivo dos cirurgiões dentistas a esses pacientes, uma parcela deles negligencia o acompanhamento odontológico. Dessa forma, esse trabalho teve como objetivo identificar o conhecimento dos profissionais de Odontologia da atenção primária à saúde no município de Quixadá-CE, frente ao tratamento odontológico de pacientes hipertensos e diabéticos.

\section{MÉTODO}

O presente estudo é uma pesquisa transversal, de campo, de caráter quantitativo. Participaram da pesquisa Cirurgiões-Dentistas (CD) da Estratégia de Saúde da Família (ESF) da sede e dos distritos do município de QuixadáCeará, localizada a aproximadamente $160 \mathrm{~km}$ da Capital do Ceará, Fortaleza. Todos os procedimentos de coleta iniciaram logo após a aprovação pelo Comitê de Ética do Centro Universitário Católica de Quixadá (parecer $\mathrm{n}^{\circ}$ 2.490.305), sendo que todos os sujeitos assinaram o Termo de Consentimento Livre e Esclarecido. A amostra não probabilística, por conveniência, foi constituída por 15 dentistas, de ambos os sexos, com idade média de 33 anos. 
Os participantes advêm do quadro de funcionários da Estratégia de Saúde da Família do Município de Quixadá-CE. Para participar do estudo, os dentistas deveriam estar trabalhando no referido município pelo menos um ano, estar cadastrado no Cadastro Nacional de Estabelecimentos de Saúde (CNES), e estar frequentando e participando das atividades odontológicas nas unidades de saúde. Foram excluídos aqueles que não estiveram nas unidades de saúde no período de aplicação do questionário, seja por período de férias, licença saúde ou outro motivo.

A realização do estudo foi autorizada pela Secretaria da Saúde do município, através da assinatura da carta de Anuência. A coleta dos dados foi realizada entre os meses de março e abril de 2019 através de um questionário estruturado (APÊNDICE I), auto preenchido com questões de múltipla escolha em língua portuguesa, na qual foram obtidas informações sobre idade, sexo, tempo de atuação, aspectos relacionados ao atendimento odontológico a pacientes hipertensos e diabéticos e sobre o autoconhecimento da conduta a ser seguida quanto ao atendimento a esses pacientes. Para cada um desses cenários, foram elaboradas afirmativas que contemplam os conhecimentos necessários para o reconhecimento da situação que é avaliar o nível de conhecimento sobre o tema proposto. $\mathrm{O}$ tempo estimado para responder as nove questões era aproximadamente de cinco minutos.

O instrumento foi validado por dois docentes da disciplina de Saúde Coletiva para discussão da temática e redação dos itens elaborados. Os itens foram corrigidos e modificados de acordo com as sugestões dos professores e novamente apresentados para apreciação final. Após obter a versão final do instrumento, este foi aplicado a um grupo composto por quatro cirurgiõesdentistas da atenção primária de faixa etária variada, sendo dois participantes da sede do município e dois dos distritos. O cálculo do Kappa apresentou um valor de 0,81 .

A análise estatística dos dados foi realizada por meio da análise descritiva com distribuição da frequência relativa e absoluta, através o programa estatístico Statiscal Package for Social Sciences (SPSS) versão 25.0 com recursos de tabelas.

\section{RESULTADOS}

Na Tabela 1 estão representados o perfil dos 15 participantes dos quais $86,7 \%(n=13)$ era do sexo feminino. Quanto a faixa etária dos participantes presentes $66,7 \%$ foi $\leq 33(n=10)$. Em relação ao perfil profissional, o tempo de atuação mais prevalente foi o de $\leq 9$ anos, representando $66,7 \%(n=10)$ do total da amostra. 
Tabela 1 - Distribuição dos voluntários por sexo, idade e tempo de atuação na odontologia

\begin{tabular}{c|c|c|c}
\hline \multicolumn{2}{c|}{ Variável } & $\mathbf{N}$ & \% \\
\hline \multirow{2}{*}{ Idade (em anos) } & $\leq 33$ & 10 & 66,7 \\
\cline { 2 - 4 } & $>33$ & 5 & 33,3 \\
\hline \multirow{2}{*}{ Sexo } & Feminino & 13 & 86,7 \\
\cline { 2 - 4 } & Masculino & 2 & 13,3 \\
\hline \multirow{2}{*}{ Tempo de formação (em anos) } & $\leq 9$ & 10 & 66,7 \\
\cline { 2 - 4 } & $>9$ & 5 & 33,3 \\
\hline
\end{tabular}

Fonte: Autores, 2019.

Por intermédio dos dados coletados, foi observado que $100 \%(n=15)$ realizam o acompanhamento odontológico com pacientes hipertensos e diabéticos. Em relação à frequência de acompanhamento desses pacientes, $40 \%(n=6)$ dos participantes realizam de 1 a 3 meses, já $60 \%(n=9)$ optam por fazer de 4 a 6 meses. Quando questionados sobre a motivação/incentivo do acompanhamento odontológico dos pacientes hipertensos e diabéticos os cirurgiões dentistas que responderam positivamente representam $100 \%$ $(n=15)$ da amostra. Os exames laboratoriais complementares para realização do atendimento dos pacientes hipertensos e diabéticos não são solicitados por $46,7 \%(n=7)$. A comunicação com o médico que acompanha esses pacientes é optada por $86,7 \%(n=13)$ dos cirurgiões-dentistas que fizeram parte do presente estudo. Observou-se também que $93,3 \%(n=14)$ realizam aferição da pressão dos pacientes em questão. A melhora na qualidade de vida dos pacientes hipertensos e diabéticos que realizam acompanhamento odontológico é observada por $93,3 \%$ $(\mathrm{n}=14)$ dos cirurgiões-dentistas da Atenção Primária de Quixadá. A resistência dos pacientes hipertensos e diabéticos para o acompanhamento odontológico é observada por $46,7 \%(n=7)$ dos participantes. As dificuldades encontradas para realizar o tratamento/acompanhamento dos pacientes hipertensos e diabéticos são relatadas por $46,7 \%(n=7)$ dos entrevistados (TABELA 2).

Tabela 2 - Características das práticas de atendimento odontológico à hipertensos e diabéticos da amostra geral.

\begin{tabular}{|c|c|c|c|}
\hline \multicolumn{2}{|l|}{ Variável } & $\mathbf{N}$ & $\%$ \\
\hline \multirow{2}{*}{ Realiza acompanhamento odontológico } & Sim & 15 & 100 \\
\hline & Não & 0 & 0 \\
\hline \multirow{2}{*}{$\begin{array}{l}\text { Frequência de acompanhamento (em } \\
\text { meses) }\end{array}$} & 1 a 3 & 6 & 40 \\
\hline & 4 a 6 & 9 & 60 \\
\hline
\end{tabular}




\begin{tabular}{|c|c|c|c|}
\hline \multicolumn{2}{|l|}{ Variável } & $\mathbf{N}$ & $\%$ \\
\hline \multirow{2}{*}{$\begin{array}{c}\text { Motiva/incentiva o acompanhamento } \\
\text { odontológico }\end{array}$} & Sim & 15 & 100 \\
\hline & Não & 0 & 0 \\
\hline \multirow{2}{*}{ Solicita exames complementares } & Sim & 8 & 53,3 \\
\hline & Não & 7 & 46,7 \\
\hline \multirow{2}{*}{ Comunica-se com o médico } & Sim & 13 & 86,7 \\
\hline & Não & 2 & 13,3 \\
\hline \multirow{2}{*}{ Realiza aferição da pressão nos pacientes } & Sim & 14 & 93,3 \\
\hline & Não & 1 & 6,7 \\
\hline \multirow{2}{*}{$\begin{array}{l}\text { Melhora na qualidade de vida dos } \\
\text { pacientes acompanhados }\end{array}$} & Sim & 14 & 93,3 \\
\hline & Não & 1 & 6,7 \\
\hline \multirow{2}{*}{$\begin{array}{l}\text { Resistência por parte dos pacientes } \\
\text { acompanhados }\end{array}$} & Sim & 7 & 46,7 \\
\hline & Não & 8 & 53,3 \\
\hline \multirow{2}{*}{$\begin{array}{l}\text { Dificuldades para realizar o tratamento e } \\
\text { acompanhamento }\end{array}$} & Sim & 9 & 60,0 \\
\hline & Não & 6 & 40,0 \\
\hline
\end{tabular}

Fonte: Autores, 2019.

\section{DISCUSSÃO}

A faixa etária predominante dos entrevistados foi de $\leq 33$ anos equivalendo a $66,7 \%(\mathrm{n}=10)$ da amostra, corroborando com nosso estudo, Frichembruder, Hugo e Hilgert (2017), observaram em seu estudo que 34,6\% dos participantes tinham idade entre 30 a 39 anos. Oshima et al. (2018), também encontraram valores semelhantes de participantes entre 26 a 35 anos de idade contabilizando $40 \%$ dos participantes do estudo.

Rodrigues, Pinheiro e Araújo (2015), em um estudo realizado sobre a percepção de acadêmicos de Odontologia sobre os seus conhecimentos para o atendimento odontológico de hipertensos e diabéticos, contabilizou também como maior parte da amostra o sexo feminino $68 \%$. Porém, um equilíbrio entre ambos os sexos foi observado no estudo de Frichembruder; Hugo e Hilgert (2017), no qual apresentou $45,2 \%$ do sexo feminino.

O perfil profissional da amostra relacionada ao tempo de formação é representado por $66,7 \%(n=10)$ de cirurgiões dentistas que atuam em uma média de $\leq 9$ anos. Já no estudo de Oshima et al. (2018), foi observado apenas $20 \%$ nessa mesma perspectiva de idade de atuação. Enquanto para Fabris et al. (2018), 33,5\% tinham de 1 a 5 anos atuando, percentual representado por maior parte da amostra.

A participação do CD na ESF, com a finalidade de realizar a promoção de saúde bucal dos usuários do serviço público, bem como a prevenção e recuperação de problemas acarretados pela ausência de um acompanhamento 
odontológico da população dependente, proporcionou uma melhoria bastante perceptível, tendo em vista que outrora esses serviços não eram oferecidos, deixando os usuários em uma situação precária em relação a saúde bucal. As unidades de ESF trouxeram à população uma melhoria de vida, através da oferta de serviços complementares e necessários para uma qualidade de vida por completo. $\mathrm{O} C D$ fica em horário comercial com toda a equipe, uma realidade inovadora para os usuários (BRASIL, 2016).

As equipes de ESF estão preparadas para oferecer à população um atendimento destinado à promoção, proteção e recuperação da saúde em nível de atenção primária. São atividades desenvolvidas pelas equipes multiprofissionais, responsáveis pelo desenvolvimento de atividades voltadas para cada público, em que os idosos contam com um acompanhamento e assistência especial que priorizam a qualidade de vida (MOTTA; AGUIAR; CALDAS, 2011).

A diabetes mellitus (DM) é caracterizada principalmente em três tipos: diabetes mellitus tipo 1, tipo 2 e diabetes gestacional. Caracteriza-se do tipo 1 a que possui destruição crônica das células pancreáticas através do mecanismo de defesa do corpo. Essa doença apresenta ausência da secreção de insulina e possui participação genética. Já a do tipo 2 é adquirida e diagnosticada quando se tem resistência periférica à insulina, em que posteriormente acontece a diminuição da secreção da mesma, ou seja, caracteriza-se como não insulino dependente. A diabetes gestacional, que geralmente ocorre no $3^{\circ}$ trimestre de gestação, caracteriza-se pela insuficiência na produção adicional de insulina devido às alterações hormonais que provocam resistência. Surge, pela primeira vez, na gestação, podendo permanecer ou não após o parto (FERREIRA; CAMPOS, 2014).

Tanto para os profissionais da saúde quanto para o paciente, o controle da DM é considerado complexo. Entretanto, para que melhores resultados sejam alcançados, o paciente precisa adquirir um estilo de vida adequado, associado a uma dieta balanceada e, como complemento, praticar exercícios físicos. Assim, é possível garantir ótimos resultados quando comparado ao tratamento farmacológico (COSTA et al., 2011).

Uma das maiores causas da morbimortalidade no mundo é ocasionada pela Hipertensão Arterial Sistêmica (HAS), gerando um enorme problema para a saúde pública, pois sua ocorrência afeta um número elevado da população. No Brasil, a doença afeta mais de $50 \%$ da população idosa e entre $15 \%$ e $20 \%$ da população adulta. A associação da doença ao uso do tabaco pode gerar um grande fator de risco para desenvolver doenças cardiovasculares, as quais podem levar o paciente a óbito, em $30 \%$ dos casos. Para que um indivíduo seja considerado hipertenso, a média de sua pressão arterial deve se manter, frequentemente, acima de 140 por $90 \mathrm{mmHg}$, segundo o Ministério da Saúde (BRASIL, 2006; FILHA; NOGUEIRA; VIANA, 2011). 
Atualmente, a HAS é denominada como uma síndrome multifatorial, pois se trata de uma doença crônica degenerativa de natureza multifatorial, de característica assintomática, na maioria dos casos, e tem seu desenvolvimento lento e progressivo. A pressão é elevada no interior dos vasos sanguíneos, devido ao comprometimento dos vasodilatadores e vasoconstritores. Esse mecanismo pode gerar lesões em órgãos nobres como o coração, rins, olhos e cérebro. A doença também é predisponente a complicações como Acidente Vascular Encefálico (AVE), Infarto Agudo do Miocárdio (IAM), insuficiência cardíaca e insuficiência renal (LESSA, 2010).

Conforme Gonçalves e Ramos (2010), a inserção de cirurgiões dentistas na Atenção Primária à Saúde é de primordial importância para que se possa acrescentar na melhoria de vida dos usuários, pois possibilita uma troca de experiências entre os profissionais de saúde que faz o acompanhamento dos pacientes que buscam atendimento, possibilitando uma promoção de saúde mais eficaz. Quando avaliadas as características da percepção dos cirurgiões dentistas da Atenção Primária à Saúde sobre seus conhecimentos para o atendimento odontológico de hipertensos e diabéticos, 100\% $(n=15)$ dos participantes relataram realizar o acompanhamento dos pacientes em questão. Corroborando com nosso estudo, Macêdo et al. (2018), obteve o mesmo resultado, $100 \%$ de sua amostra que também realiza o acompanhamento odontológico desses pacientes.

A acessibilidade aos serviços da atenção básica necessita de dedicação da equipe para que o usuário se sinta motivado a retornar. Conforme Ribeiro et al. (2015), o planejamento adequado nas unidades, levando em consideração a sua estrutura física, proporcionam melhor qualidade e acessibilidade para os pacientes. Em nosso estudo, $60 \%(n=9)$ dos cirurgiões dentistas atendiam o retorno dos pacientes hipertensos e diabéticos entre 4 a 6 meses após cada consulta. Para Gonçalves e Ramos (2010), mesmo a atenção básica tendo o propósito de aproximar a equipe dos usuários, isso ainda não garante a utilização de forma contínua pelos pacientes.

Assis, Da Silva Simões e Cavalcanti (2012), Dias e Campos (2012), propõem que o envelhecimento da população juntamente ao processo de urbanização da população resultou na incidência de doenças crônicas degenerativas, sendo a hipertensão arterial sistêmica e diabetes mellitus as que ocorrem com maior frequência na sociedade. Com essa mudança no perfil epidemiológico brasileiro concomitante aos avanços de políticas públicas de saúde possibilitou visar o controle das doenças citadas através de programas preventivos e reorganização dos serviços públicos, pois foi notória essa necessidade, observada através de estudos estatísticos. Um dos meios de obter sucesso na prevenção é incentivar o retorno para as consultas periódicas. No presente estudo, a motivação/incentivo é efetuada por 100\% dos participantes.

A solicitação de exames complementares foi um dos assuntos que apresentou resultados próximos, sendo estes solicitados por $53,3 \%(n=8)$ dos 
cirurgiões dentistas. Pilger; Menon e De Freitas Mathias (2013), observaram em seu estudo que esses exames foram realizados por 30,5\% do sexo masculino enquanto $69,5 \%$ não realizaram, enquanto o sexo feminino mostrou um resultado menos negativo, onde $42,9 \%$ realizaram e $57,1 \%$ não. Já Rodrigues; Pinheiro e Araújo (2015) apresentaram em seu estudo que 92,6\% solicitam exames laboratoriais.

A comunicação com os médicos que acompanham os pacientes hipertensos e diabéticos é relatada por $86,7 \%(n=13)$ do total da amostra. Conforme Gonçalves e Ramos (2010), o mecanismo de troca de experiências entre os profissionais da atenção básica é capaz de gerar uma grande melhoria na atenção básica se inserir e adaptar a equipe à realidade de cada usuário, com suas prioridades e necessidades específicas. Pequenos atos do cotidiano auxiliam também para uma melhoria, sendo que através deles é possível uma análise onde uma nova representação à assistência possa articular os profissionais, a gestão e a população, realizando uma mesma ação que é a promoção de saúde. Lopes et al. (2015), acredita que a falta de comunicação entre a equipe de profissionais resulta na falta de qualificação do cuidado do usuário, assim, impossibilitando utilizar as diversas oportunidades de atenção à saúde.

A realização da aferição da Pressão Arterial (PA) dos pacientes é praticada por $93,3 \%(n=14)$ dos entrevistados. Em contra partida, Rodrigues; Pinheiro e Araújo (2015) obtiveram resultado distinto, onde pouco mais da metade, 52,6\% afere a PA dos pacientes previamente ao atendimento. Observação semelhante no estudo de De Souza Schueroff; De Oliveira Peres e Barbosa (2016), que dentre todas as mulheres que participaram da pesquisa, 50\% aferem a pressão dos pacientes em todos os atendimentos e $30 \%$ aferem apenas em procedimentos cirúrgicos. Porém, 41,7\% afirmaram aferir apenas em procedimentos cirúrgicos ou pacientes hipertensos e 20,83\% aferem independente do procedimento.

A melhora na qualidade de vida dos pacientes hipertensos e diabéticos que realizam acompanhamento odontológico na Atenção Básica à Saúde é observada por 93,3\% (n=14). Para Motta; Aguiar e Caldas (2011), a avaliação da qualidade dos serviços à saúde é uma das ferramentas mais importantes para o planejamento adequado, proporcionando uma melhoria significativa. Ribeiro et al. (2015), ainda acrescenta que quando é realizado o planejamento correto através do que tem disponível nas estruturas físicas da Atenção Básica, proporcionam uma melhor qualidade ao atendimento e consequentemente uma melhora na vida dos usuários que buscam atendimentos nos estabelecimentos de saúde.

A resistência dos pacientes hipertensos e diabéticos para $\mathrm{o}$ acompanhamento odontológico é observada por $46,7 \%(n=7)$. Porém, para Pilger; Menon e De Freitas Mathias (2013) observou-se que a maior demanda de pacientes dos serviços de saúde é composta pela população idosa, resultado do 
aumento da longevidade. Fernandes; Bartoli e Barros (2009) acrescenta ainda que os serviços de saúde são mais frequentados pelo sexo feminino.

As dificuldades para realizar o tratamento e/ou acompanhamento dos pacientes hipertensos e diabéticos foi relatada por $60 \%(n=9)$ dos cirurgiões dentistas. Macêdo et al. (2018), observaram que $80 \%$ dos cirurgiões dentistas realizavam acompanhamento aos pacientes com necessidades especiais. Motta; Aguiar e Caldas (2011), relatam também que a educação em saúde proporciona um melhor vínculo entre o profissional e o paciente, bem como o ambiente social e físico, além de proporcionar uma melhor qualidade de vida.

$\mathrm{O}$ estudo realizado apresentou limitações importantes quanto à sua população e amostra, que ao se apresentar em número reduzido, permite considerar os resultados encontrados apenas para a população em questão.

\section{CONCLUSÃO}

Diante dos resultados obtidos no presente estudo observou-se que os cirurgiões-dentistas da Atenção Primária à Saúde de Quixadá-CE apresentam um bom conhecimento frente ao tratamento odontológico dos pacientes hipertensos e diabéticos, pois todos sabem da importância de realizar o acompanhamento desses usuários do serviço de saúde, bem como o incentivo para que esses busquem o acesso periódico do tratamento. Porém, quando relacionado a solicitação de exames complementares e comunicação com o médico que acompanham os pacientes, foi detectada a necessidade de uma capacitação aos profissionais para promover a padronização desses pontos. Já para os pacientes é necessária proporcionar ações de promoção da saúde a fim de demonstrar a importância desses atendimentos para uma melhor qualidade de vida, possibilitando a redução da resistência e dificuldades para uma continuidade dos serviços de saúde bucal.

\section{REFERÊNCIAS}

ALBUQUERQUE, Adriana Bezerra Brasil de; BOSI, Maria Lúcia Magalhães. Visita domiciliar no âmbito da Estratégia Saúde da Família: percepções de usuários no Município de Fortaleza, Ceará, Brasil. Cadernos de Saúde Pública, v. 25, n. 5, p.1103$12,2009$.

ALVES, Davi Barroso; BARBOSA, Maria Tereza Serrano. Desigualdades na mortalidade por doenças crônicas entre idosos e sua associação com indicadores socioeconômicos no Brasil. Revista Brasileira de Ciências do Envelhecimento Humano, v.7, n.1, p. 22-33, 2010.

ASSIS, Luana Couto; DA SILVA SIMÕES, Mônica Oliveira; CAVALCANTI, Alessandro Leite. Políticas públicas para monitoramento de hipertensos e diabéticos na atenção básica, Brasil. Revista Brasileira de Pesquisa em Saúde/Brazilian Journal of Health Research, p. 65-70. 2012. 
BACKES, Dirce Stein et al. Vínculo profissional usuário: competência para a atuação na Estratégia Saúde da Família. Avances en Enfermería, Florianópolis. v. 33, n. 2, p. 222-229. 2015.

BRASIL. Ministério da Saúde (MS). Secretaria de Atenção à Saúde. Departamento de Atenção Básica. Envelhecimento e Saúde da Pessoa Idosa. Brasília: MS; 2006.

Secretaria de Atenção à Saúde. Departamento de Atenção Básica. Cadernos de Atenção Básica, nº 16 (Série A); p.56, 2016.

FILHA, Francidalma Soares Sousa Carvalho; NOGUEIRA, Lídya Tolstenko; VIANA, Lívia Maria Mello. Hiperdia: Adesão e percepção de usuários acompanhados pela Estratégia de Saúde da Família. Revista de Rede de Enfermagem do Nordeste, Fortaleza, v.12, p. 930-936, 2011.

CHAVES, Sônia Cristina Lima et al. Política Nacional de Saúde Bucal: fatores à integralidade do cuidado. Revista de Saúde Pública, v.44, n.6, p. 1005-1013. Out-2010.

COSTA, Jorge de Assis et al. Promoção da saúde e diabetes: discutindo a adesão e a motivação de indivíduos diabéticos participantes de programas de saúde. Ciência \& Saúde Coletiva, Rio de Janeiro, v. 16, n. 3, p. 2001-2009, Mar. 2011.

DE SOUZA SCHUEROFF, Edgar; DE OLIVEIRA PERES, Marcos Vinicius; BARBOSA, Carmem Patrícia. Importância do conhecimento do cirurgião-dentista sobre pressão arterial, fatores modificadores e complicações sistêmicas durante atendimento cirúrgico. Arquivos do MUDI, v,20, n.3, p.44-58. 2016.

DIAS, Juliana Chioda Ribeiro; CAMPOS, Juliana Alvares Duarte Bonini. Diabetes mellitus: razão de prevalência nas diferentes regiões geográficas no Brasil, 2002 2007. Ciência \& Saúde Coletiva, Rio de Janeiro v. 17, n. 1, 2012.

FABRIS, Vínicius et al. Conhecimento dos cirurgiões dentistas sobre o uso de anestésicos locais em pacientes: diabéticos, hipertensos, cardiopatas, gestantes e com hipertireoidismo. Journal of Oral Investigations, Passo Fundo, vol. 7, n. 1, p. 3351, Jan.-Jun., 2018.

FERNANDES, Léia Cristiane L.; BERTOLDI, Andréa D.; BARROS, Aluísio JD.

Utilização dos serviços de saúde pela população coberta pela Estratégia de Saúde da Família. Revista de saúde pública, v. 43, n. 4, p. 595-603, 2009.

FERREIRA, Valceir Aparecido; CAMPOS, S. M. B. Avanço Farmacológico No Tratamento do Diabetes Tipo 2. Brazilian Journal of Surgery and Clinical Research, v. 8, n. 3, p. 72-78, Paraná. 2014.

FRICHEMBRUDER, Karla; HUGO, Fernando Neves; HILGERT, Juliana Balbinot. Perfil e percepção de profissionais dos Centros de Especialidades Odontológicas do Rio Grande do Sul. Revista da Faculdade de Odontologia-UPF, Passo Fundo, v. 22, n. 1, p. 43-48, jan./abr. 2017. 
GOMES, Marília Miranda Forte et al. Associação entre mortalidade e estado marital: uma análise para idosos residentes no Município de São Paulo, Brasil, Estudo

SABE, 2000 e 2006. Cadernos de Saúde Pública, v. 29, n. 3, p. 566-78, 2013.

GONÇALVES, Evelise Ribeiro; RAMOS, Flávia Regina Souza. O trabalho do cirurgião-dentista na estratégia de saúde da família: potenciais e limites na luta por um novo modelo de assistência. Interface-Comunicação, Saúde, Educação, v.14, n. 33, p.301-314, 2010.

ILHA, Silomar et al. Vínculo profissional-usuário em uma equipe da Estratégia

Saúde da família. Ciência, Cuidado e Saúde. Rio Grande. v. 13, n. 3, p. 556-562, 2014.

LESSA, I. Hipertensão arterial sistêmica no Brasil: tendência temporal. Cadernos de Saúde Pública, Rio de Janeiro, v. 26, n. 8, ago. 2010.

LOPES, Adriana Santos et al. O acolhimento na Atenção Básica em saúde: relações de reciprocidade entre trabalhadores e usuários. Saúde em Debate, Rio de Janeiro, v. 39, n. 104, p. 114-123, Jan/Mar, 2015.

MACÊDO, Giulian Lennon et al. Acesso ao atendimento odontológico dos pacientes especiais: a percepção de cirurgiões-dentistas da atenção básica. Revista Ciência Plural, v. 4, n. 1, p. 67-80, 2018.

MIYAMOTO, Adriana Yuriko et al. Qualidade de vida de idosos em uma instituição de longa permanência. Arquivos de Ciências da Saúd, v. 23, p. 36-40, 2016.

MOTTA, Luciana Branco da; AGUIAR, Adriana Cavalcanti de; CALDAS, Célia Pereira. Estratégia Saúde da Família e a atenção ao idoso: experiências em três municípios brasileiros. Cadernos de Saúde Pública, v. 27, n. 4, p. 779-786, 2011.

OSHIMA, Ana Marcia Mieko Yamaoka et al. Perfil, atuação e satisfação de cirurgiões-dentistas em Residências Multiprofissionais em Saúde da região Sul do Brasil. Revista da ABENO, v. 18, n. 1, p. 134-145, 2018.

PILGER, Calíope; MENON, Mario Umberto; DE FREITAS MATHIAS, Thais Aidar. Utilização de serviços de saúde por idosos vivendo na comunidade. Revista da Escola de Enfermagem da USP, v. 47, n. 1, p. 213-20, 2013.

RIBEIRO, Juliana Pires et al. Acessibilidade aos serviços de saúde na Atenção Básica do Estado de Goiás. Revista Eletrônica de Enfermagem. v. 17, n. 3, jul./ set. 2015.

RODRIGUES, Kédma Pureza; PINHEIRO, Helder Henrique Costa; ARAÚJO, Marizeli Viana de Aragão. Percepção de acadêmicos de Odontologia sobre seus conhecimentos para o atendimento odontológico de hipertensos e diabéticos. Revista da ABENO, v. 15, n. 4, p. 19-28, 2015. 


\section{APÊNDICE I - QUESTIONÁRIO}

\section{PERCEPÇÃO DOS CIRURGIÕES-DENTISTAS DA ATENÇÃO PRIMÁRIA A SAÚDE SOBRE SEUS CONHECIMENTOS PARA O ATENDIMENTO ODONTOLÓGICO DE HIPERTENSOS E DIABÉTICOS}

Idade: Sexo: ( ) Feminino ( ) Masculino Tempo de atuação:

01. Realiza acompanhamento odontológico com pacientes hipertensos e diabéticos? 1.SIM ( ) 2.NÃO ( )

02. Com qual frequência realiza o acompanhamento desses pacientes?

1 a 3 meses ( ) 4 a 6 meses ( ) NÃO ACOMPANHO ( )

03. Solicita exames laboratoriais complementares para atende-los? 1.SIM ( ) 2.NÃO ( )

04. Comunica-se com o médico que acompanha o paciente? 1.SIM ( ) 2.NÃO ( )

05. Realiza aferição da pressão nos pacientes? 1.SIM ( ) 2.NÃO ( )

06. Você observa alguma melhora na qualidade de vida dos pacientes hipertensos e diabéticos que realizam acompanhamento odontológico?

1.SIM ( ) 2.NÃO ( ) 4.NÃO ACOMPANHO ( )

07. Você observa alguma resistência desses pacientes para realizar o acompanhamento odontológico?

$1 . S I M(\quad)$ 2.NÃO ( )

08. Você sente dificuldades para realizar o tratamento e/ou acompanhamento dos pacientes hipertensos e diabéticos?

1.SIM ( ) 2.NÃO ( ) 4.NÃO ACOMPANHO ( )

A discussão precisa ser revisada pensando em evitar a fragmentação que esta parte do manuscrito apresenta.

O primeiro parágrafo trata das características da amostra, o segundo, abruptamente, já passa a falar sobre diabetes em uma sequência interessante que contempla a discussão sobre as doenças crônicas.

O sétimo parágrafo retoma as características da amostra, seguindo com a participação do CD na ESF citando as especificidades do atendimento na saúde da família que pode ser relacionado como mais adequados às características das doenças crônicas elencadas anteriormente. $\mathrm{O}$ décimo parágrafo se refere a Visita domiciliar, mas que não foi abordada em nenhum lugar do texto anteriormente. Pode servir como exemplo das atividades da ESF, mas talvez este seja um parágrafo dispensável.

Depois a discussão segue com questões relacionadas á melhoria da qualidade de vida que não deve ser considerada a partir da pergunta, talvez seja melhor focar a discussão na percepção dos profissionais sobre a possibilidade de melhoria da qualidade de vida do que inferir que a qualidade de vida modifica a partir da percepção dos profissionais, que é o que a pergunta procura verificar. 